\title{
Silicone-induced granuloma of breast implant capsule mistaken for breast implant-associated anaplastic large cell lymphoma
}

\author{
Yun-Seob Kim ${ }^{1}$, Si-Gyun Roh $^{1,2}$, \\ Nae-Ho Lee ${ }^{1,2}$, Jin-Yong Shin ${ }^{1,2}$ \\ ${ }^{1}$ Department of Plastic and \\ Reconstructive Surgery, Jeonbuk National \\ University Medical School, Jeonju; \\ ${ }^{2}$ Research Institute of Clinical Medicine of \\ Jeonbuk National University-Biomedical \\ Research Institute of Jeonbuk National \\ University Hospital, Jeonju, Korea
}

For patients with breast implants who present with an isolated seroma, capsular contracture, or peri-implant mass, clinicians must rule out breast implant-associated anaplastic large cell lymphoma (BIA-ALCL). As non-malignant silicone-induced granuloma of breast implant capsule (SIGBIC) may mimic BIA-ALCL, particular care must be exercised to prevent misdiagnosis. In this report, we describe three cases of SIGBIC misdiagnosed as BIA-ALCL. In each of these cases, a preoperative evaluation including breast magnetic resonance imaging and physical examination, as well as the fact that a textured silicone implant was used in the patients, indicated a high probability of malignancy. In all three cases, however, an explorative operation and pathologic results revealed foreign body granulomas without malignant features. While it is critical that potential cases of BIA-ALCL be diagnosed and treated quickly, hasty judgments may lead to misdiagnosis and severe emotional distress in patients. Increased awareness of SIG$\mathrm{BIC}$, specifically its mimicry of BIA-ALCL, may be helpful to avoid these outcomes.

Keywords Anaplastic large cell lymphoma / Breast implant / Foreign-body granuloma / Case reports

\section{INTRODUCTION}

Breast implant-associated anaplastic large cell lymphoma (BIAALCL) has recently attracted clinicians' attention. Unlike other primary breast lymphomas, which develop from B cells, BIA-ALCL is a $\mathrm{T}$ cell-derived lymphoma [1,2]. Its typical onset time is 3 to 7 years after mammoplasty [3], and it presents as an isolated seroma, capsular contracture, or peri-implant mass [4]. Since it was first reported in 1997 by Keech and Creech [5], increasingly many cases have been confirmed.

Received: Sep 24, 2021 Revised: Nov 16, 2021 Accepted: Nov 19, 2021 Correspondence: Si-Gyun Roh Department of Plastic and Reconstructive Surgery, Jeonbuk National University Hospital, 20 Geonji-ro, Deokjin-gu, Jeonju 54907, Korea

Tel: +82-63-250-1860, Fax: +82-63-250-1866, E-mail: pssroh@jbnu.ac.kr

Copyright $\odot 2022$ The Korean Society for Aesthetic Plastic Surgery.

This is an Open Access article distributed under the terms of the Creative Commons Attribution Non-Commercial License (https://creativecommons.org/licenses/by-nc/4.0/) which permits unrestricted non-commercial use, distribution, and reproduction in any medium, provided the original work is properly cited. www.e-aaps.org
In suspected BIA-ALCL patients, it is important to rule out the possibility of malignancy and diagnose/treat the patient without delay. Excessively hasty judgments, however, lead to misdiagnosis and severe emotional distress to patients and families. A cautious approach should be taken to prevent this undesirable event. Silicone-induced granuloma of breast implant capsule (SIGBIC) is often mistaken for BIA-ALCL, as its radiologic and clinical features are very similar. Particular attention is needed in these cases $[4,6]$.

In this report, we describe three cases in which SIGBIC was mistaken for BIA-ALCL. In each case, the clinical history and manifestation mimicked the malignant tumor. In all cases, there was a lack of evidence available to distinguish between the two disease entities, and the diagnosis was only possible based on a histologic examination. These considerations may play an important role in future cases.

\section{CASE REPORT}

Case 1

A 53-year-old woman visited our hospital with upward displace- 
ment of the left breast (Fig. 1). She had undergone augmentation mammoplasty 29 years ago, and the left breast deformity occurred 6 months earlier. Breast magnetic resonance imaging (MRI) was performed, and the radiologist suggested the possibility of BIA-ALCL with extracapsular rupture (Fig. 2).

The authors planned an exploration to remove the implant and obtain histologic confirmation. Intraoperatively, we found the implant in the subpectoral plane, and the capsule was full of bloody/ serous fluid. The implant was a textured silicone implant, and the breast capsule showed an unusual morphology containing multiple nodules inside (Fig. 3). The peri-implant fluid and capsule were examined for histologic confirmation.

A cytological examination of the fluid showed that there were no malignant cells, but inflammatory cells were present. The capsule tissue was diagnosed as a foreign body granuloma lacking the

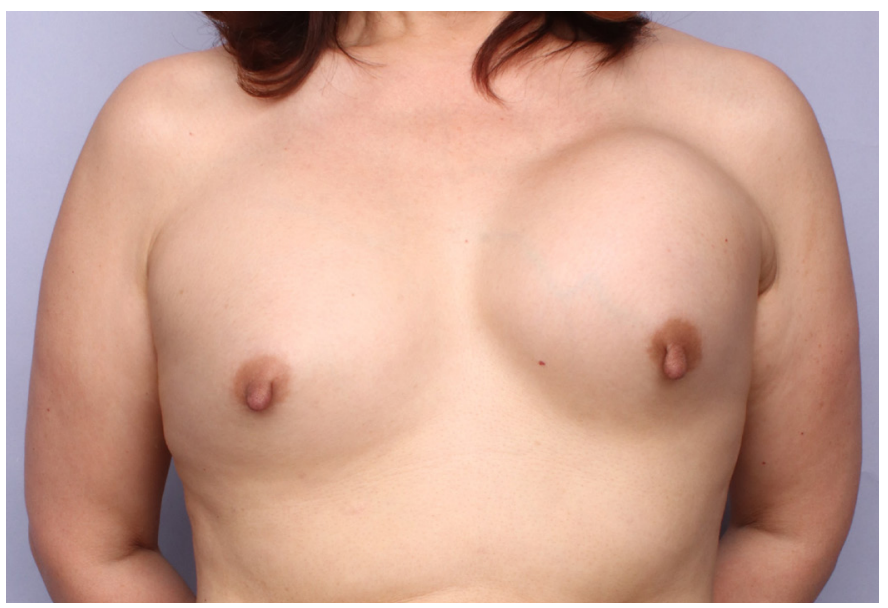

Fig. 1. Preoperative photograph in case 1 showing upward positioning of the left breast.

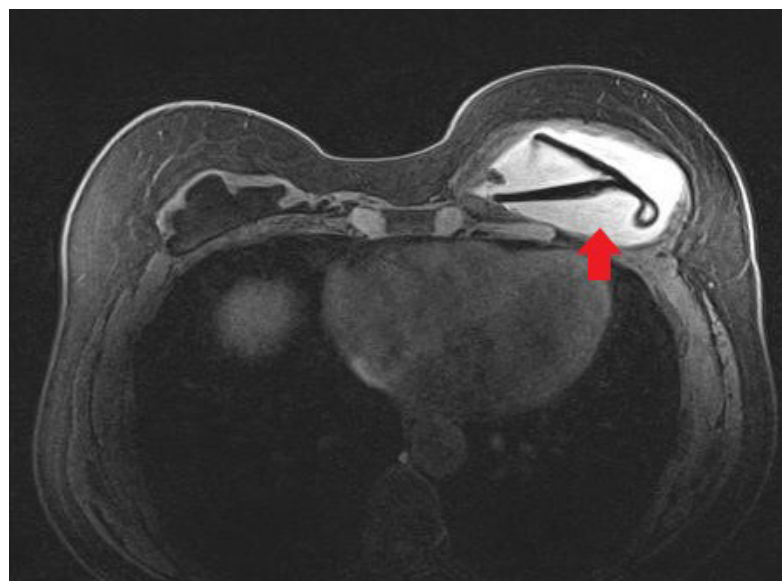

Fig. 2. Breast magnetic resonance image in case 1. The left breast implant is ruptured and peri-implant fluid collection (red arrow) is seen with a thickened capsule wall. malignant features of BIA-ALCL, including CD30 positivity (Fig. 4). After the operation, the patient did not complain of any discomfort.

\section{Case 2}

A 42-year-old woman presented with sudden swelling of her right breast after removal of both implants at a local clinic 1 week prior (Fig. 5). Recurrent edematous changes took place in her right breast starting 2 years ago, and the local doctor predicted that her symptoms were caused by implant rupture. Her prosthesis was 17 years old and silicone-textured. Indeed, her right implant had ruptured. However, after the first operation, her right breast suddenly became swollen and she was referred to our hospital. Breast MRI revealed a mass-like lesion with high signal intensity in the right subareolar area and an enlarged right axillary lymph node. This was interpreted as BIA-ALCL with a high likelihood of late hematoma.

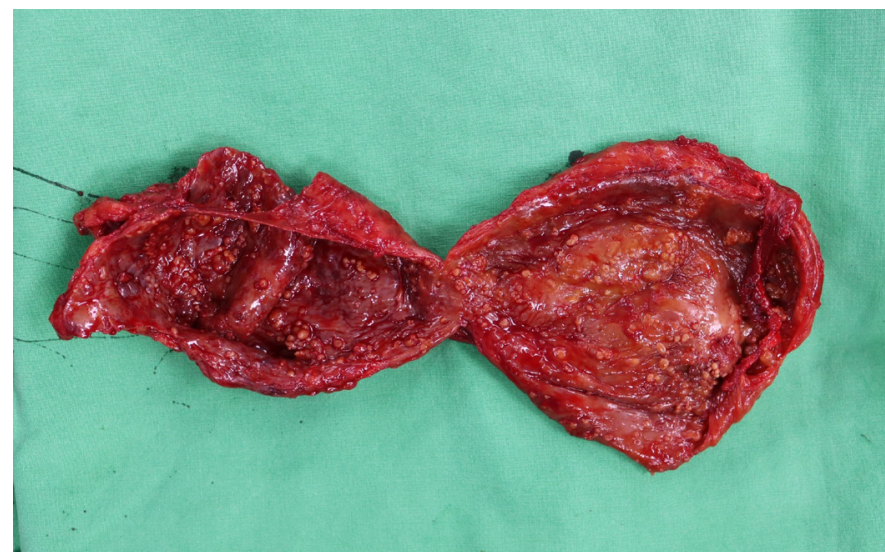

Fig. 3. The breast capsule removed in case 1. The inner surface showed irregular nodularity.

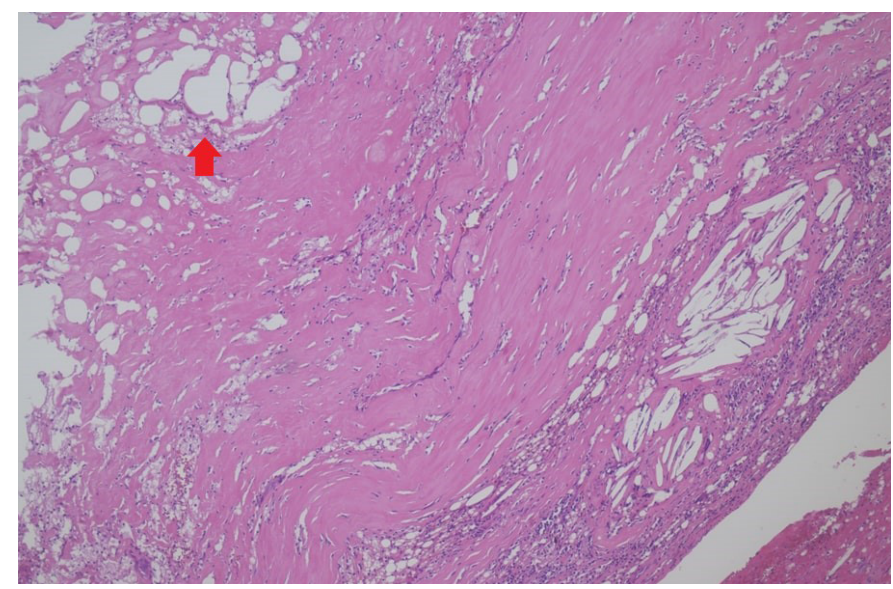

Fig. 4. Microscopic view of the breast implant capsule in case 1. Numerous empty cystic vacuoles with focal fibrous rims around the vacuoles (red arrow) $(H \& E, \times 200)$. 


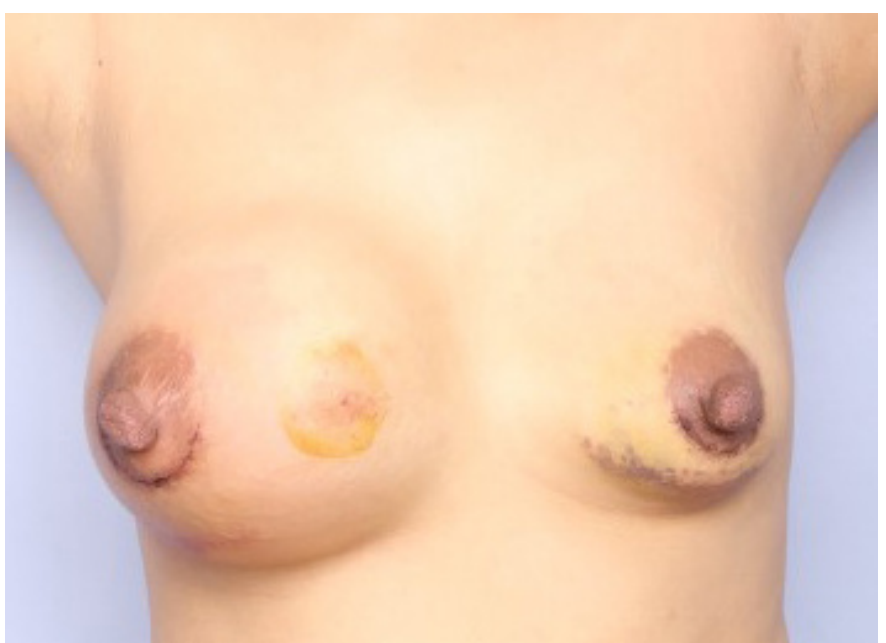

Fig. 5. Preoperative photograph in case 2 showing the swollen right breast.

We approached the lesion through the right inframammary fold line with a periareolar incision, at which point a large amount of dark-red opaque fluid poured out. Inside, we found dense and fibrotic remnants of the implant capsule that were difficult to completely evacuate.

A microscopic examination showed fibrotic tissues with chronic inflammation and late hematoma. Cytology showed a combination of neutrophils and red blood cells. The patient's right breast remained swollen afterward; therefore, a secondary evacuation was performed. After studying the microscopic appearance, the lesion turned out to be benign.

\section{Case 3}

A 47-year-old patient was diagnosed with a left breast implant rupture 5 years after mammoplasty. Revisional mammoplasty with the insertion of Mentor MemoryGel silicone-filled smooth breast implants (Style 7000 Smooth, Smooth Round Moderate Classic Profile Gel-Filled Breast Implant, $170 \mathrm{cc}$ ) was done on her left breast. The previously inserted implant was silicone-textured. Approximately 9 months later, a volumetric change presenting as capsular contracture occurred in her left breast (Fig. 6). Preoperative MRI revealed capsular contracture with a suspicious mass-like lesion in the left upper quadrant. However, the prosthesis remained intact this time.

The surgical intervention revealed severe contracture of the implant capsule. After the operation, a biopsy was performed, and the histologic results showed an inflammatory response without malignant transformation.

\section{DISCUSSION}

The etiopathogenesis of BIA-ALCL is still under investigation. How-

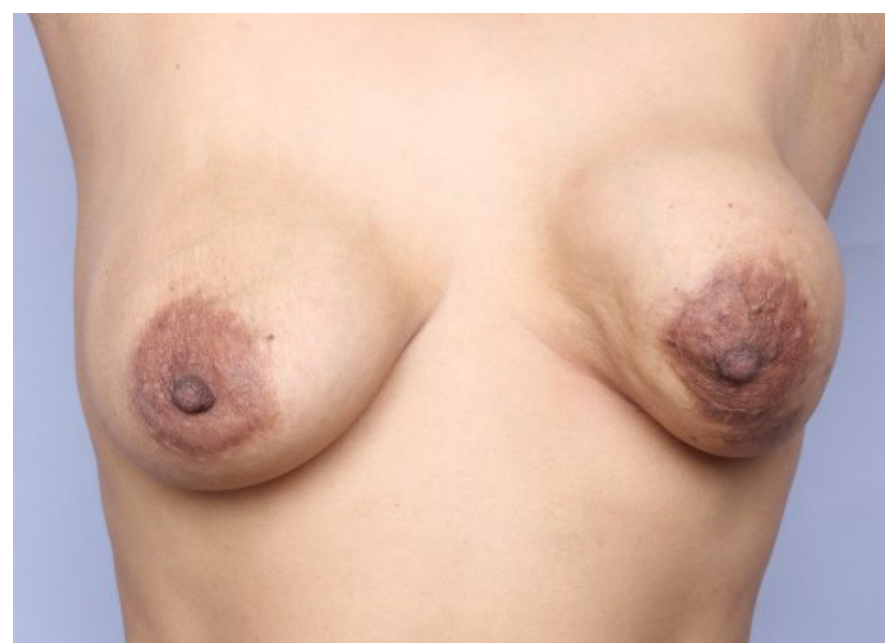

Fig. 6. Preoperative photograph in case 3 with the left breast shrunken due to capsular contracture.

ever, recent studies have suggested that the immune response induced by silicone particles from the implant may play an important role [7]. Leakage of silicone into the capsule can occur not only in ruptured implants, but even in those which appear grossly intact [4]. These foreign bodies from the prosthesis cause an immune over-reaction with chronic activation of $\mathrm{T}$ lymphocytes and monoclonal neoplasia [8]. The etiology of SIGBIC is therefore similar to that of BIA-ALCL, although in the former case, the chronic activation of $\mathrm{T}$ cells does not lead to monoclonal hyperplasia $[9,10]$. Accordingly, a microscopic differential diagnosis is possible, as the former condition lacks anaplastic monoclonal $\mathrm{T}$ cells enhanced by CD30 or CD3 immunostaining [8].

The signs and symptoms of the two diseases are very similar [11]. Patients usually present with seroma formation without spontaneous resolution, capsular contracture, or a peri-implant mass. On imaging studies, intracapsular fluid with or without implant rupture may be visible [12]. In most cases, the gold standard of treatment is explantation and eradication of the fibrous capsule [13]. When these symptoms appear with solid masses or distant metastasis, additional treatment including a secondary operation, chemotherapy, or radiation may be needed $[13,14]$.

In conclusion, BIA-ALCL and SIGBIC are both rare and difficult to distinguish from each other. To prevent unnecessary anxiety and worry among patients, and to ensure that the most well-suited treatments are recommended, clinicians should accordingly be familiar with both conditions.

\section{NOTES}

\section{Conflict of interest}

No potential conflict of interest relevant to this article was reported. 


\section{Ethical approval}

The study was approved for exemption by the Institutional Review Board of Jeonbuk National University Hospital (IRB exemption No. CUH 2021-09-045).

\section{Patient consent}

The patients provided written informed consent for the publication and the use of their images.

\section{ORCID}

Yun-Seob Kim

Si-Gyun Roh

Nae-Ho Lee

Jin-Yong Shin

https://orcid.org/0000-0002-4443-6434

https://orcid.org/0000-0003-2865-0075

https://orcid.org/0000-0003-1354-8203

https://orcid.org/0000-0003-2680-7411

\section{REFERENCES}

1. Hwang MJ, Brown H, Murrin R, et al. Breast implant-associated anaplastic large cell lymphoma: a case report and literature review. Aesthetic Plast Surg 2015;39:391-5.

2. Jeanneret-Sozzi W, Taghian A, Epelbaum R, et al. Primary breast lymphoma: patient profile, outcome and prognostic factors. A multicentre Rare Cancer Network study. BMC Cancer 2008;8:86.

3. Roden AC, Macon WR, Keeney GL, et al. Seroma-associated primary anaplastic large-cell lymphoma adjacent to breast implants: an indolent T-cell lymphoproliferative disorder. Mod Pathol 2008;21:455-63.

4. Fleury EF, Rego MM, Ramalho LC, et al. Silicone-induced granuloma of breast implant capsule (SIGBIC): similarities and differences with anaplastic large cell lymphoma (ALCL) and their differential diagnosis. Breast Cancer (Dove Med Press) 2017;9:133-40.

5. Keech JA Jr, Creech BJ. Anaplastic T-cell lymphoma in proximity to a saline-filled breast implant. Plast Reconstr Surg 1997;100:554-5.

6. Shepard E, Kamenko S, Snir OL, et al. Silicone granuloma mimicking breast implant associated large cell lymphoma (BIA-ALCL): a case report. Case Reports Plast Surg Hand Surg 2020;7:63-7.

7. Cohen Tervaert JW, Kappel RM. Silicone implant incompatibility syndrome (SIIS): a frequent cause of ASIA (Shoenfeld's syndrome). Immunol Res 2013;56:293-8.

8. de Faria Castro Fleury E, D’Alessandro GS, Lordelo Wludarski SC. Silicone-induced granuloma of breast implant capsule (SIGBIC): histopathology and radiological correlation. J Immunol Res 2018;2018: 6784971.

9. Castro C, Fernandes D, Mendonca M, et al. Silicone-induced granuloma of breast implant capsule mimicking anaplastic large cell lymphoma. Breast J 2020;26:1028-30.

10. Fleury EF. Synchronous breast implant associated anaplastic large cell lymphoma (BIA-ALCL) and silicone induced granuloma of breast implant capsule (SIGBIC): what to learn. Radiol Case Rep 2020;15:173642.

11. Carson B, Cox S, Ismael H. Giant siliconoma mimicking locally advanced breast cancer: a case report and review of literature. Int J Surg Case Rep 2018;48:54-60.

12. de Faria Castro Fleury E, Gianini AC, Ayres V, et al. Breast magnetic resonance imaging: tips for the diagnosis of silicone-induced granuloma of a breast implant capsule (SIGBIC). Insights Imaging 2017;8:43946.

13. Kim B, Roth C, Young VL, et al. Anaplastic large cell lymphoma and breast implants: results from a structured expert consultation process. Plast Reconstr Surg 2011;128:629-39.

14. Talwalkar SS, Miranda RN, Valbuena JR, et al. Lymphomas involving the breast: a study of 106 cases comparing localized and disseminated neoplasms. Am J Surg Pathol 2008;32:1299-309. 